OPEN ACCESS

Edited by:

Jihui Zhang,

The Chinese University of

Hong Kong, China

Reviewed by:

Yaping Liu,

The Chinese University of

Hong Kong, China

Huijuan Wu,

Second Military Medical

University, China

Xiangdong Tang,

Sichuan University, China

*Correspondence:

Zan Wang

wangzan@jlu.edu.cn;

wangzanprof@163.com

Yi Yang

yang_yi@jlu.edu.cn;

doctoryangyi@163.com

†These authors share first authorship

Specialty section:

This article was submitted to

Sleep Disorders,

a section of the journal

Frontiers in Neurology

Received: 28 November 2020

Accepted: 16 February 2021

Published: 11 March 2021

Citation:

Deng F, Zhang $Y$, Zhang $R$, Tang $Q$,

Guo $Z$, Lv Y, Wang $Z$ and Yang $Y$

(2021) Compromised Dynamic

Cerebral Autoregulation in Patients

With Central Disorders of

Hypersomnolence.

Front. Neurol. 12:634660

doi: 10.3389/fneur.2021.634660

\section{Compromised Dynamic Cerebral Autoregulation in Patients With Central Disorders of Hypersomnolence}

\author{
Fang Deng ${ }^{1+}$, Yanan Zhang ${ }^{1+}$, Ran Zhang ${ }^{1}$, Qi Tang ${ }^{1}$, Zhenni Guo ${ }^{2}$, Yudan Lv ${ }^{1}$, Zan Wang ${ }^{1 *}$ \\ and Yi Yang ${ }^{2 *}$ \\ ${ }^{1}$ Department of Neurology, The First Hospital of Jilin University, Changchun, China, ${ }^{2}$ Department of Neurology, Clinical Trial \\ and Research Center for Stroke, The First Hospital of Jilin University, Changchun, China
}

Objective: We aimed to investigate the dynamic cerebral autoregulation (dCA) in patients with central disorders of hypersomnolence during wakefulness.

Methods: Thirty-six patients with central disorders of hypersomnolence were divided into three groups according to polysomnography and multiple sleep latency test results: the idiopathic hypersomnia group $(\mathrm{H})$, narcolepsy type 1 without rapid-eye-movement sleep behavior disorder group (NT1-RBD), and narcolepsy type 1 with rapid-eye-movement sleep behavior disorder group (NT1 + RBD), with 12 patients in each group. Twelve sex- and age-matched healthy controls were recruited. We assessed the Epworth sleepiness scale (ESS) and dCA of all subjects. dCA was assessed by analyzing the phase difference (PD) using transfer function analysis. The ESS and dCA were analyzed before and after standardized treatment in 24 patients with narcolepsy type 1.

Results: The overall PD of the $\mathrm{H}, \mathrm{NT1}-\mathrm{RBD}$, and NT1 + RBD groups were lower than that of the control group $(P<0.001)$. There were no significant differences between the overall PD of the NT1-RBD and NT1 + RBD group $(P>0.05)$. The ESS scores decreased and the overall PD increased after treatment in 24 patients with narcolepsy type $1(P<$ 0.001). Multivariable analysis showed that mean sleep latency in multiple sleep latency test was independently associated with impaired overall PD $(P<0.05)$.

Conclusions: The dCA is impaired in patients with central disorders of hypersomnolence. The impairment of dCA occurs irrespective of NT1-RBD/+RBD. The ESS score and dCA improved in patients with narcolepsy type 1 after medication treatment. The mean sleep latency in multiple sleep latency test was independently associated with impaired dCA.

Clinical Trial Registration: www.ClinicalTrials.gov, identifier: NCT02752139.

Keywords: central disorders of hypersomnolence, narcolepsy type 1, idiopathic hypersomnia, rapid-eye-movement sleep behavior disorder, dynamic cerebral autoregulation 


\section{INTRODUCTION}

The central disorders of hypersomnolence include narcolepsy type 1 (NT1), narcolepsy type 2 (NT2), and idiopathic hypersomnia (IH) (1). Studies have demonstrated a decreased cerebral blood flow (CBF) in patients with NT1 and IH (2, 3 ), which implies that cerebral autoregulation (CA) function may play an important role in the central disorders of hypersomnolence. CA is a physiological mechanism of the brain that maintains sufficient CBF despite changes in the blood or cerebral perfusion pressure. Dynamic cerebral autoregulation (dCA) can respond to real-time changes in the blood pressure within seconds, allowing continuous measurement of CA and a wave by wave analysis of hemodynamics (4). Many diseases may impair $\mathrm{dCA}$, including cerebrovascular diseases, traumatic brain injury and Alzheimer's disease $(5,6)$. The dCA mechanism is complex; it includes myogenic, metabolic, endothelial, and neurogenic mechanisms, and neurogenic including the monoaminergic and cholinergic mechanisms (7). Studies have found the monoaminergic mechanism including 5hydroxytryptamine and norepinephrine to be implicated in the central disorders of hypersomnolence $(8,9)$, which are vasoactive substances and likely affect the dCA $(10,11)$.

Rapid-eye-movement sleep behavior disorder (RBD) affects $30-63 \%$ of NT1 patients (12). Idiopathic RBD exhibits decreased cerebral perfusion and dCA $(13,14)$; however, there are currently no studies on RBD and dCA in NT1 patients. We hypothesize that $\mathrm{dCA}$ is impaired in patients with central disorders of hypersomnolence. We obtained dCA data from patients with IH and NT1 and investigated the relationship between central disorders of hypersomnolence and dCA.

\section{METHODS}

The study was approved by the ethics committee of the First Hospital of Jilin University and followed the guidelines of the Declaration of Helsinki (1964). Written informed consent was obtained from all the participants or their relevant guardians.

\section{Subjects}

Patients with central disorders of hypersomnolence, that underwent the polysomnography (PSG) and multiple sleep latency tests (MSLT), were recruited from the Department of Neurology, First Hospital of Jilin University, from March 2018 to March 2019. A total of 36 patients were enrolled with central disorders of hypersomnolence [12 patients with IH, 12 with NT1 without RBD group (NT1-RBD), and 12 with NT1 with RBD group (NT1 + RBD), respectively]. Twelve age- and sex-matched healthy controls were also recruited from the same region, based on their PSG results.

\section{Inclusion Criteria}

The inclusion criteria for the different groups were as follows: (1) Control group: age- and sex-matched healthy volunteers without sleep disorder in PSG; (2) NT1-RBD and NT1 + RBD groups: subjective sleepiness, cataplexy, mean sleep latency $\leq 8 \mathrm{~min}$, and $\geq 2$ sleep-onset rapid-eye-movement (REM) periods (SOREMP) on MSLT or one SOREMP on the preceding night PSG coupled with one SOREMP on the MSLT. The diagnostic criteria of RBD was based on the International Classification of Sleep Disorders 3rd edition; (3) IH group: subjective sleepiness, absence of cataplexy, mean sleep latency $\leq 8 \mathrm{~min}$, and with one time or without SOREMP (including no SOREMP on the PSG from the preceding night); (4) the age cut-off value being 15 to 65 ; and (5) good bilateral temporal window penetration.

\section{Exclusion Criteria}

The exclusion criteria were as follow: presence of (1) sleeprelated breathing disorders, circadian rhythm disorders, and other causes of disturbed nocturnal sleep; (2) history of secondary daytime sleepiness, epilepsy, mental illness, or drug abuse; (3) arrhythmia, hyperthyroidism, and other hemodynamic factors; (4) intracranial and extracranial vascular stenosis or occlusion diagnosed by vascular ultrasound; and (5) not cooperating with the questionnaire survey.

\section{Collection of Clinical Data}

All subjects underwent a comprehensive collection of general and clinical data, including age, sex, medical history, Epworth sleeping scale (ESS) evaluation, neurological examination, and head magnetic resonance imaging.

\section{Polysomnography}

All the subjects were monitored for at least $8 \mathrm{~h}$ in the sleep center of our hospital using PSG (Compumedics, Australia). The PSG results were analyzed by professional sleep technicians, that had PSG technologist certification, by referring to the revised interpretation criteria of sleep stages and related events issued by the American Academy of Sleep Medicine version 2.1.

\section{Multiple Sleep Latency Tests}

After PSG recording, the central electroencephalogram (C3$\mathrm{A} 2, \mathrm{C} 4-\mathrm{A} 1)$ and occipital electroencephalogram (O1-A2, O2A1), left and right electrooculogram, electromyography, and electrocardiography were retained. The first nap was performed 2-3 h after the PSG examination. Afterward, 4-5 naps were taken at intervals of $2 \mathrm{~h}$. Activities were avoided $15 \mathrm{~min}$ before each test. Sleep was avoided during the $2 \mathrm{~h}$ interval. A total of $15 \mathrm{~min}$ were recorded after falling asleep, including the $20 \mathrm{~min}$ before falling asleep. If only one SOREMP was recorded in the first four naps, an additional 5th nap was also recorded.

\section{Dynamic Cerebral Autoregulation Measurement}

The participants were instructed to avoid alcohol, nicotine, and caffeine intake, and exercise for at least $12 \mathrm{~h}$ before the measurement. The measurement was performed in a quiet, dedicated research laboratory. First, the subjects had their baseline arterial blood pressure (Omron 711) and heart rates measured (15). Then beat-to-beat arterial blood pressure and continuous bilateral middle cerebral artery blood flow velocity were recorded for $10 \mathrm{~min}$. The measurement data were then stored for further dynamic cerebral autoregulation examination analysis (16). 
TABLE 1 | Clinical characteristics in the patients with IH, NT1-RBD, NT1 + RBD, and controls.

\begin{tabular}{|c|c|c|c|c|c|}
\hline & Controls $(n=12)$ & IH $(n=12)$ & NT1-RBD $(n=12)$ & NT1 + RBD $(n=12)$ & $P$-value \\
\hline Gender (male/female) & $8 / 4$ & $8 / 4$ & $7 / 5$ & $8 / 4$ & 0.965 \\
\hline Age (years) & $31.9 \pm 7.9$ & $39.8 \pm 13.9$ & $29.4 \pm 10.3$ & $34.9 \pm 13.8$ & 0.177 \\
\hline Mean ABP (mmHg) & $83.3 \pm 6.1$ & $87.9 \pm 5.0$ & $84.7 \pm 5.8$ & $83.3 \pm 5.3$ & 0.061 \\
\hline Heart rate (bmp) & $71.5 \pm 6.2$ & $70.9 \pm 10.0$ & $69.8 \pm 7.7$ & $70.2 \pm 5.6$ & 0.944 \\
\hline End-tidal $\mathrm{CO}_{2}(\mathrm{mmHg})$ & $36.6 \pm 3.6$ & $36.7 \pm 4.0$ & $37.0 \pm 3.9$ & $36.6 \pm 2.6$ & 0.990 \\
\hline
\end{tabular}

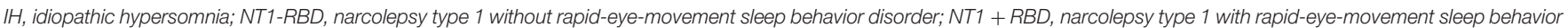
disorder; $A B P$, arterial blood pressure.

\section{Analysis of Dynamic Cerebral Autoregulation}

Recorded data were processed using the MATLAB software (Math Works, Natick, MA, USA). The data analysis of dCA was performed using the transfer function analysis (TFA) (15). TFA between the arterial blood pressure and cerebral blood flow velocity was calculated as the quotient of the cross-spectrum of the two signals and the auto spectrum of arterial blood pressure in the low-frequency domain $(0.06-0.12 \mathrm{~Hz})$ to obtain the frequency-dependent estimates of phase difference (PD), where the derived parameters are considered to be the most relevant to autoregulation hemodynamics (17). A decreased $\mathrm{PD}$ represents impaired dCA. Coherence was calculated to estimate the reliability of the relationship between the two signals at the frequency domain, and the later statistical analysis was performed only if the coherence of the parameters was $>0.5$ (18).

\section{Statistical Analysis}

The SPSS 23.0 software was used for statistical analysis. The Shapiro-Wilk test was used to assess the normal distribution of continuous variables. Measurement data with a normal distribution [including ESS, $\mathrm{PD}$, age, mean arterial blood pressure $(\mathrm{ABP})$, heart rate, and end-tidal $\mathrm{CO}_{2}$ ] were expressed as mean and standard deviation whereas data with skewed distribution were expressed as median (interquartile range). Categorical data (gender) were expressed as absolute values. Paired $t$-test was used to compare the differences between the two paired samples; ESS and PD in patients with NT1, before and after treatment. One-way ANOVA or Kruskal-Willis $\mathrm{H}$ test was used to compare the differences between the multiple groups of independent samples (ESS and PD of IH, NT1-RBD, NT1 $+\mathrm{RBD}$, and control groups) based on the data distribution. Univariate and multivariate linear regression were used to assess the association of PD and clinical parameters including the total sleep time (TST), mean sleep latency in MSLT, sleep efficiency, percent of stage 1 non-REM (NREM) time in TST (stage N1), percent of stage 2 NREM time in TST (stage N2), percent of stage 3 NREM time in TST (stage N3), percent of REM sleep time in TST (REM sleep), and arousal index. In the post-hoc analysis, the Bonferroni method was used to calculate the adjusted $P$-value. $P$-values below 0.05 were considered statistically significant.

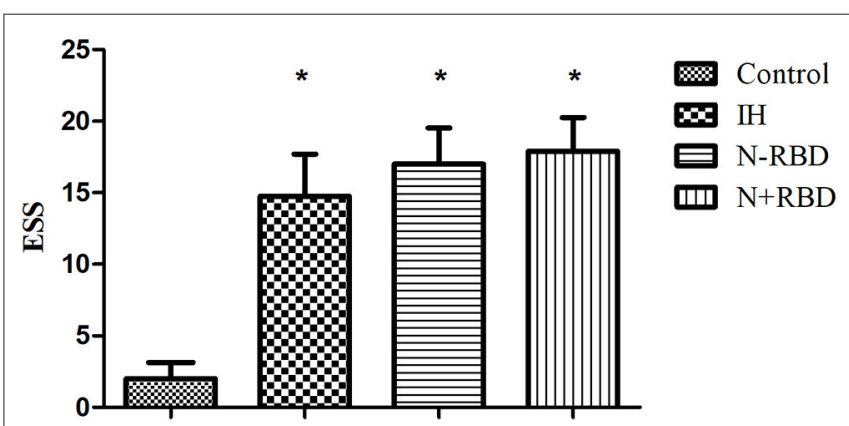

FIGURE 1 | ESS scores in the patients with IH, NT1-RBD, NT1 + RBD, and controls. ${ }^{*}$ Different from controls $(P<0.05)$. ESS, Epworth sleepiness scale.

\section{RESULTS}

\section{Baseline Characteristics}

In total, 48 participants were enrolled in this study, including 12 IH patients, 12 NT1-RBD patients, 12 NT1 + RBD patients, and 12 controls. There are no significant differences in gender, age, mean $\mathrm{ABP}$, heart rate, and end-tidal $\mathrm{CO}_{2}$ among all the groups. The clinical characteristics of the participants are described in Table 1.

\section{ESS Score}

ESS was used to assess the degree of sleepiness. The ESS scores of patients in the IH, NT1-RBD, and NT1 + RBD groups (14.8 \pm $3.0,17.0 \pm 2.5,17.9 \pm 2.4)$ were higher than those of the control group $(2.1 \pm 1.1, P<0.001)$ (Figure 1).

\section{dCA Parameters}

The overall PD of patients in the IH, NT1-RBD, and NT1 + RBD groups $\left(34.36 \pm 6.51,35.96 \pm 8.99,32.90 \pm 9.98^{\circ}\right)$ was significantly lower than that of the control group (52.98 \pm $6.33^{\circ}, P<0.001$ ) (Figure 2); however, there were no significant differences between the overall PD of the NT1-RBD and NT1 + RBD group (35.96 \pm 8.99 vs. $\left.32.90 \pm 9.98^{\circ}, P>0.05\right)$.

\section{ESS and dCA Parameters of NT1 Before and After Treatment}

Among 24 patients with NT1, the ESS and dCA were examined after 1 -month treatment with methylphenidate $(18 \mathrm{mg}$, once a day in the morning) and venlafaxine ( $75 \mathrm{mg}$, once a day in the 

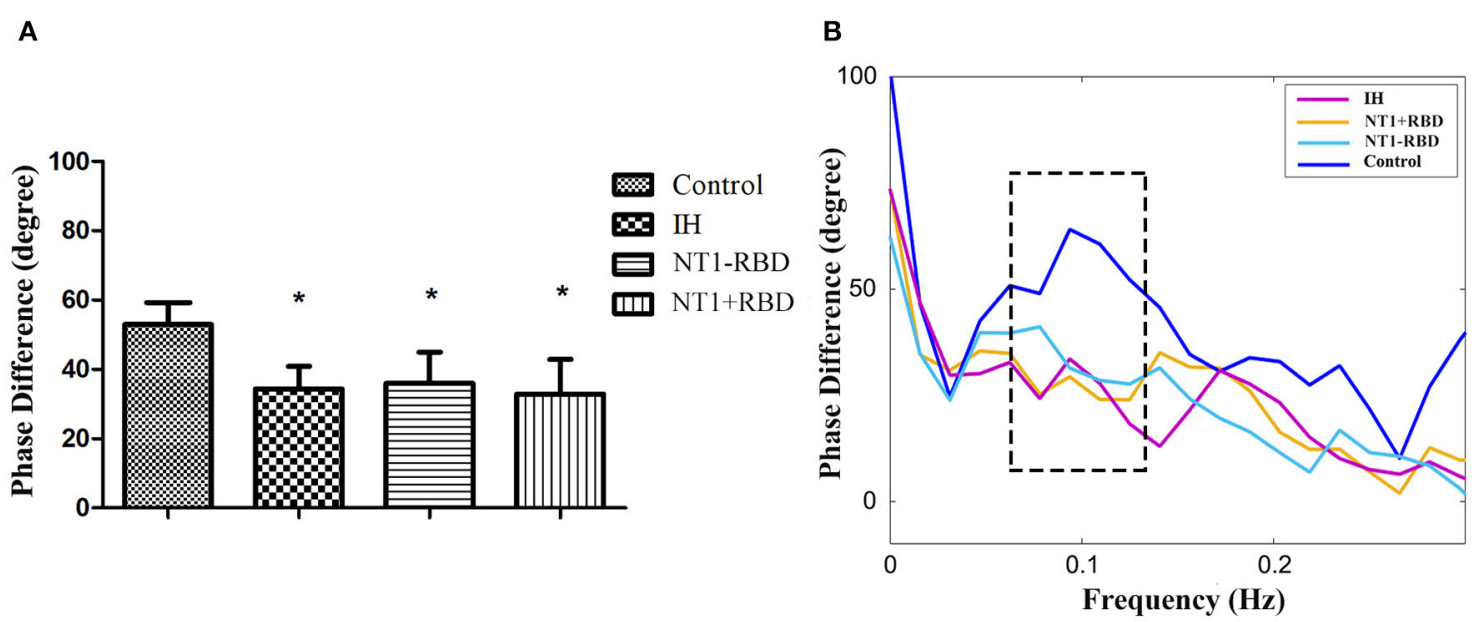

FIGURE 2 | The autoregulatory parameter and statistical distributions in the patients with IH, NT1-RBD, NT1 + RBD, and controls. Statistical distributions of overall phase difference $\mathbf{( A )}$ and its transfer function $\mathbf{( B )}$ in each group. *Difference of overall phase difference in patients with IH, NT1-RBD, NT1 + RBD, and controls $(P<0.05)$.

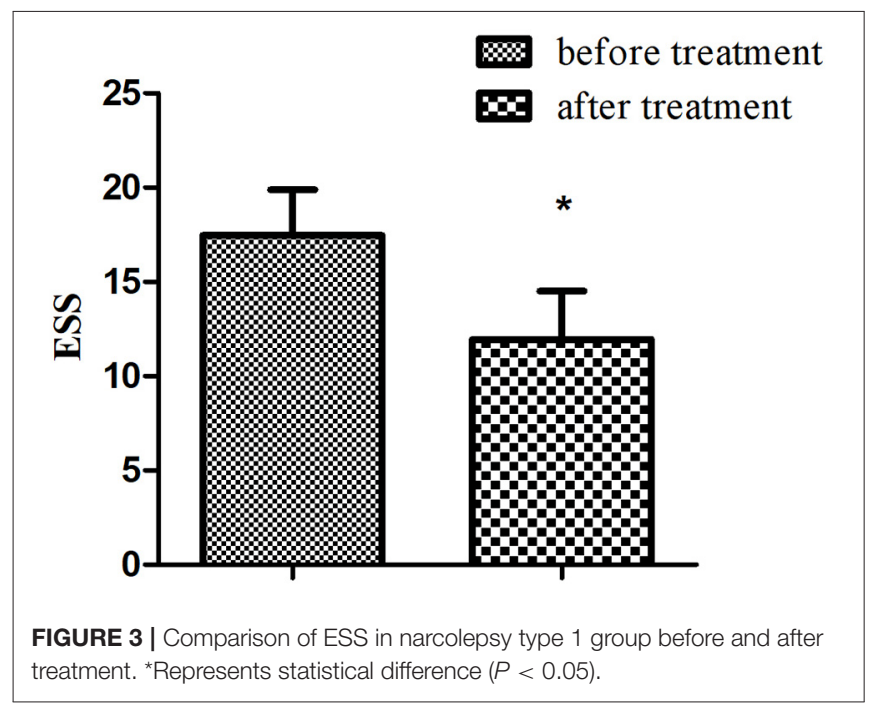

morning). The ESS score decreased (11.96 \pm 2.55 vs. $17.46 \pm$ 2.43, $P<0.001$ ) (Figure 3 ) and the overall PD increased after the treatment ( $47.37 \pm 9.31$ vs. $34.43 \pm 9.42^{\circ}, P<0.001$ ) (Figure 4).

\section{Univariable and Multivariable Analyses}

The clinical parameters used in the univariable and multivariable analysis are shown in Table 2 . In the univariable model, the overall PD correlated with mean sleep latency in MSLT $(P<$ $0.001)$, sleep efficiency $(P<0.05)$, stage N1 $(P<0.05)$, and arousal index $(P<0.05)$. The multivariable model included mean sleep latency in MSLT, sleep efficiency, stage N1, stage N2, stage N3, and arousal index. Mean sleep latency in MSLT $(P<$ $0.05)$ was an independent factor that influenced the overall PD (Table 2).

\section{DISCUSSION}

We found the cerebral autoregulatory parameter compromised in patients with central disorders of hypersomnolence. There were no significant differences in the degree of impairment between the NT1-RBD and NT1 + RBD patients. The ESS score and dCA improved after medication for the NT1 patients. The mean sleep latency in MSLT was independently associated with impaired dCA.

Studies have found abnormal CA in NT1 patients, evident by hypoperfusion of the hypothalamus, thalamus, prefrontal cortex, hippocampus, and cingulate gyri by single-photon emission computed tomography $(2,19)$, and abnormal CA is most likely implicated in the progression of neurological symptoms (such as cataplexy and sleep paralysis). The pathogenesis of compromised dCA in NT1 patients is the deficiency of hypocretin and monoaminergic neurons (9, 20). Hypocretin is involved in REM sleep and motor regulation through its effects on monoaminergic cells, including dopamine (DA), norepinephrine (NA), 5-hydroxytryptamine (5-HT), and other neurons $(9,21)$. NA is a sleep autonomic neuromodulating transmitter, which is an important factor for dCA (10). 5-HT is a vasoactive substance and has a potential role in dCA (11). In addition, hypocretin deficiency and a lower concentration of monoaminergic neurotransmitters lead to decreased activated projection to the basal forebrain and tuberomammillary nucleus; they are responsible for the maintenance of cortical arousal (22). Furthermore, the hypocretin neurons are located in the lateral hypothalamus and around the fornix, which projects to the regulatory centers of several autonomic nerves, including the autonomic neurons in the periaqueductal gray matter, the nucleus tractus solitarius, the nucleus ambiguous, the dorsal vagal nucleus, and the intermediolateral column of the spinal cord. Reduced or absent hypocretin levels in the inferior colliculus may reduce sympathetic excitability in NT1 patients 
A

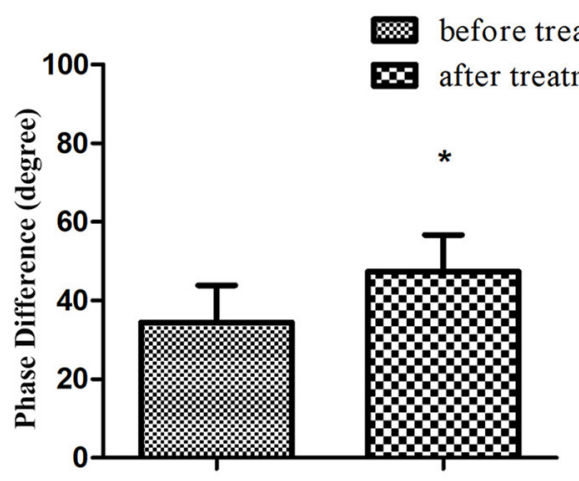

B

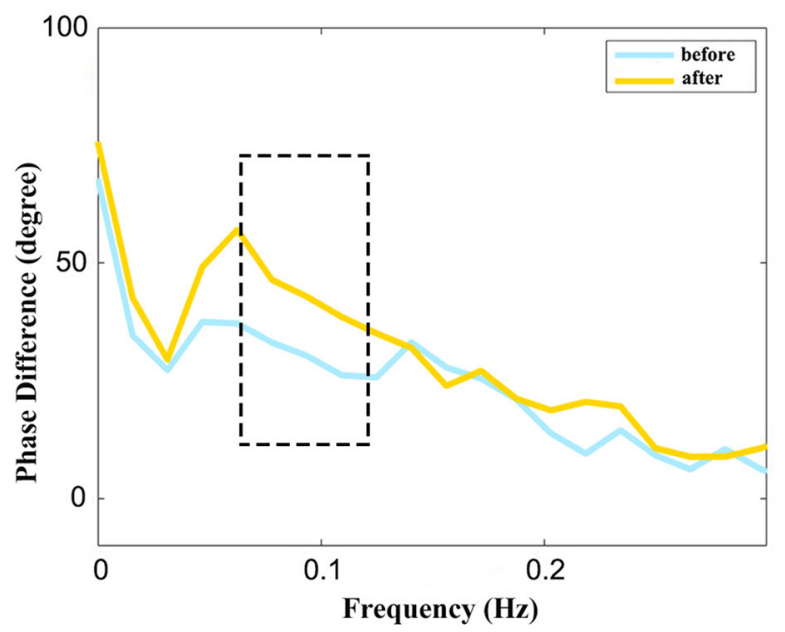

FIGURE 4 | The autoregulatory parameter and statistical distributions in narcolepsy type 1 group before and after treatment. Statistical distributions of phase difference $\mathbf{( A )}$ and its transfer function $\mathbf{( B )}$ in narcolepsy type 1 group before and after treatment. *Represents statistical difference $(P<0.05)$.

TABLE 2 | Univariable and multivariable analysis of polysomnography parameters associated with the overall phase difference.

\begin{tabular}{lcccc}
\hline Factors & \multicolumn{4}{c}{ Overall phase difference ( ${ }^{\circ}$ ) } \\
\cline { 2 - 5 } & \multicolumn{3}{c}{ Univariable analysis } & Multivariable analysis \\
\cline { 2 - 5 } & $\boldsymbol{\beta}$ & $\boldsymbol{P}$ & $\boldsymbol{P}$ \\
\hline TST (min) & 0.107 & 0.555 & & $0.016^{\mathrm{bc}}$ \\
Mean sleep latency in MSLT & 0.647 & $<0.001^{\mathrm{ab}}$ & 0.470 & \\
(min) & & & & 0.785 \\
Sleep efficiency (\%) & 0.416 & $0.016^{\mathrm{ab}}$ & 0.059 & 0.128 \\
Stage N1 (\%) & -0.559 & $0.001^{\mathrm{ab}}$ & -0.515 & 0.511 \\
Stage N2 (\%) & 0.330 & $0.060^{\mathrm{a}}$ & -0.168 & 0.379 \\
Stage N3 (\%) & 0.298 & $0.092^{\mathrm{a}}$ & 0.246 & \\
REM sleep (\%) & 0.238 & 0.183 & & 0.993 \\
Arousal index & -0.376 & $0.031^{\mathrm{ab}}$ & -0.002 & \\
\hline
\end{tabular}

TST, total sleep time; MSLT, multiple sleep latency test; REM, rapid eye movement; stage N1, percent of stage 1 non-REM (NREM) time in TST; stage N2, percent of stage 2 NREM time in TST; stage N3, percent of stage 3 NREM time in TST; REM sleep, percent of REM sleep time in TST.

${ }^{a}$ Nominally significant values $(P<0.1)$ included in the multivariable model.

${ }^{b} P$-value $<0.05$ (statistically different).

c Independent factor that influences overall phase difference.

(23). Our study showed that the dCA was impaired in NT1 patients, which may be related to hypocretin and monoamine neurotransmitter deficiency.

Narcolepsy is the second cause of RBD. Knudsen et al. found hypocretin deficiency to be independently associated with the prevalence of RBD outcomes (symptoms and muscle activity) during REM sleep in narcolepsy (24), which may explain the absence of a significant difference between the overall PD in the NT1-RBD and NT1 + RBD group; they may share the same mechanism. RBD sometimes can be the heralding symptom of NT1, forerunning the occurrence of cataplexy (25).
The pathological mechanism of $\mathrm{IH}$ is currently unclear. Studies have shown a normal concentration of hypocretin in the cerebrospinal fluid of IH patients; however, with low levels of DA, NA and 5-HT metabolites $(8,26,27)$. In addition, IH patients have autonomic symptoms (cold extremities, palpitations, and fainting episodes) (27). We hypothesize that the impaired dCA in $\mathrm{IH}$ patients may be related to the lower concentration of monoaminergic neurotransmitters.

Drug therapy for narcolepsy in this study included methylphenidate and venlafaxine. Methylphenidate is a non-competitive DA reuptake blocker, which inhibits 5-HT and NA to a certain extent. Venlafaxine is a 5-HT-NA reuptake inhibitor that increases the concentration of 5-HT and NA. After 1-month treatment with the two drugs, the symptoms of EDS and the dCA improved in patients with narcolepsy, which may be related to the increased blood concentration of 5-HT and NA, and is consistent with previous reports $(28,29)$.

The MSLT is an objective test that measures the tendency to fall asleep under controlled conditions. It is based on the notion that sleep latency reflects the underlying physiological sleepiness (30). Since the mean sleep latency is shortened in $\mathrm{IH}$ and NT1 patients on MSLT, we found the mean sleep latency in MSLT to be independently associated with the impaired dCA. The potential mechanisms are still unclear. We hypothesize the 5-HT and NA levels to be a potential mechanism that explains the underlying relationship between the mean sleep latency and the dCA; larger sample size is required to confirm this result.

Our study shows that dCA is impaired in patients with central disorders of hypersomnolence, which may involve the monoaminergic mechanism; the relationship with hypocretin remains to be explored. The compromised dCA in patients with central disorders of hypersomnolence can explain and predict the clinical symptoms, and provide a new dynamic evaluating method for central disorders of hypersomnolence. 
This study has some limitations. First, we did not examine the hypocretin of cerebrospinal fluid. Second, we did not detect the monoaminergic neurotransmitters in the participants' serum. Third, the sample size of this study is small, which may limit the analysis.

\section{CONCLUSION}

The dCA is impaired in patients with central disorders of hypersomnolence. The dCA impairment occurs irrespective of NT1-RBD/+RBD. The ESS score and dCA improved in patients with NT1 after medication. The mean sleep latency in MSLT was independently associated with impaired dCA.

\section{DATA AVAILABILITY STATEMENT}

The raw data supporting the conclusions of this article will be made available by the authors, without undue reservation.

\section{ETHICS STATEMENT}

The studies involving human participants were reviewed and approved by the Ethics Committee of the First Hospital of Jilin University. Written informed consent to participate in this study was provided by the participants' legal guardian/next of kin. Written informed consent was obtained from the individual(s),

\section{REFERENCES}

1. Khan Z, Trotti LM. Central disorders of hypersomnolence: focus on the narcolepsies and idiopathic hypersomnia. Chest. (2015) 148:26273. doi: 10.1378/chest.14-1304

2. Wada M, Mimura M, Noda Y, Takasu S, Plitman E, Honda M, et al. Neuroimaging correlates of narcolepsy with cataplexy: a systematic review. Neurosci Res. (2019) 142:16-29. doi: 10.1016/j.neures.2018.03.005

3. Boucetta S, Montplaisir J, Zadra A, Lachapelle F, Soucy JP, Gravel P, et al. Altered regional cerebral blood flow in idiopathic hypersomnia. Sleep. (2017) 40:zsx140. doi: 10.1093/sleep/zsx140

4. Claassen JA, Meel-van den Abeelen AS, Simpson DM, Panerai RB. International Cerebral Autoregulation Research Network (CARNet). Transfer function analysis of dynamic cerebral autoregulation: a white paper from the International Cerebral Autoregulation Research Network. J Cereb Blood Flow Metab. (2016) 36:665-80. doi: 10.1177/0271678X15626425

5. Donnelly J, Aries MJ, Czosnyka M. Further understanding of cerebral autoregulation at the bedside: possible implications for future therapy. Expert Rev Neurother. (2015) 15:169-85. doi: 10.1586/14737175.2015.996552

6. Claassen JA, Zhang R. Cerebral autoregulation in Alzheimer's disease. J Cereb Blood Flow Metab. (2011) 31:1572-7. doi: 10.1038/jcbfm.2011.69

7. Hamel E. Perivascular nerves and the regulation of cerebrovascular tone. J Appl Physiol. (2006) 100:1059-64. doi: 10.1152/japplphysiol.009 54.2005

8. Baruzzi A, Cirignotta F, Coccagna G, Calderini G, Lugaresi E. Cerebrospinal fluid homovanillic acid and 5-hydroxyindoleacetic acid in hypersomnia with periodic apneas or idiopathic hypersomnia: preliminary results. Sleep. (1980) 3:247-9. doi: 10.1093/sleep/3.3-4.247

9. Hagan JJ, Leslie RA, Patel S, Evans ML, Wattam TA, Holmes S. Orexin A activates locus coeruleus cell firing and increases arousal in the rat. Proc Natl Acad Sci USA. (1999) 96:10911-6. doi: 10.1073/pnas.96.19.10911

10. Armstead WM, Riley J, Vavilala MS. Norepinephrine protects cerebral autoregulation and reduces hippocampal necrosis after traumatic brain injury and minor(s)' legal guardian/next of kin, for the publication of any potentially identifiable images or data included in this article.

\section{AUTHOR CONTRIBUTIONS}

$\mathrm{FD}, \mathrm{YZ}$, and ZG wrote the manuscript. RZ conducted the data acquisition and data analysis. QT prepared the figures. YL analyzed the PSG. ZW and YY managed the study and edited the final manuscript. All authors contributed to the article and approved the submitted version.

\section{FUNDING}

The article was supported by the National Natural Science Foundation of China (Grant Number: 82071489), the Foundation of the Department of Science and Technology of Jilin Province (Grant Number: 20190201038JC), and the Foundation of Chinese Sleep Research Society Hansoh Project (Grant Number: 2019HSB05) to ZW.

\section{ACKNOWLEDGMENTS}

The authors would like to acknowledge the following individuals at Jilin University: Chunhui Wei for their technical support and Qiong Yu for the statistical analysis. via blockade of ERK MAPK and IL-6 in juvenile pigs. J Neurotrauma. (2016) 33:1761-7. doi: 10.1089/neu.2015.4290

11. Geday J, Hermansen F, Rosenberg R, Smith DF. Serotonin modulation of cerebral blood flow measured with positron emission tomography (PET) in humans. Synapse. (2005) 55:224-9. doi: 10.1002/syn.20112

12. Antelmi E, Pizza F, Franceschini C, Ferri R, Plazzi G. REM sleep behavior disorder in narcolepsy: a secondary form or an intrinsic feature? Sleep Med Rev. (2020) 50:101254. doi: 10.1016/j.smrv.2019.101254

13. Vendette M, Gagnon JF, Soucy JP, Gosselin N, Postuma RB, Tuineag $\mathrm{M}$, et al. Brain perfusion and markers of neurodegeneration in rapid eye movement sleep behavior disorder. Mov Disord. (2011) 26:171724. doi: $10.1002 / \mathrm{mds} .23721$

14. Lv S, Wang Z, Sun X, Jin H, Liu J, Deng F, et al. Compromised dynamic cerebral autoregulation in patients with idiopathic rapid eye movement behavior disorder: a case-control study using transcranial doppler. Front Psychiatry. (2020) 11:51. doi: 10.3389/fpsyt.2020.00051

15. Lv S, Guo ZN, Jin H, Sun X, Jia M, Ma H, et al. Compromised dynamic cerebral autoregulation in patients with epilepsy. Biomed Res Int. (2018) 2018:6958476. doi: 10.1155/2018/6958476

16. Luo MY, Guo ZN, Qu Y, Zhang P, Wang Z, Jin H, et al. Compromised dynamic cerebral autoregulation in patients with depression. Front Psychiatry. (2019) 10:373. doi: 10.3389/fpsyt.2019.00373

17. van Beek AH, Claassen JA, Rikkert MG, Jansen RW. Cerebral autoregulation: an overview of current concepts and methodology with special focus on the elderly. J Cereb Blood Flow Metab. (2008) 28:1071-85. doi: 10.1038/jcbfm.2008.13

18. Kuo TB, Chern CM, Sheng WY, Wong WJ, Hu HH. Frequency domain analysis of cerebral blood flow velocity and its correlation with arterial blood pressure. J Cereb Blood Flow Metab. (1998) 18:3118. doi: 10.1097/00004647-199803000-00010

19. Joo EY, Hong SB, Tae WS, Kim JH, Han SJ, Cho YW. Cerebral perfusion abnormality in narcolepsy with cataplexy. Neuroimage. (2005) 28:4106. doi: 10.1016/j.neuroimage.2005.06.019 
20. Thannickal TC, Moore RY, Nienhuis R, Ramanathan L, Gulyani S, Aldrich M. Reduced number of hypocretin neurons in human narcolepsy. Neuron. (2000) 27:469-74. doi: 10.1016/S0896-6273(00)00058-1

21. Black SW, Yamanzka A, Kilduff TS. Challenges in the development of therapeutics for narcolepsy. Progress in Neurobiology (2017) 152:89113. doi: 10.1016/j.pneurobio.2015.12.002

22. Jones BE. Arousal systems. Front Biosci. (2003) 8:s438-51. doi: 10.2741/1074

23. Chiara B, Alessandro S. The link between narcolepsy and autonomic cardiovascular dysfunction: a translational perspective. Clin Auton Res. (2018) 28:545-55. doi: 10.1007/s10286-017-0473-Z

24. Knudsen S, Gammeltoft S, Jennum PJ. Rapid eye movement sleep behaviour disorder in patients with narcolepsy is associated with hypocretin-1 deficiency. Brain. (2010) 133:568-79. doi: 10.1093/brain/awp320

25. Antelmi E, Pizza F, Vandi S, Neccia G, Ferri R, Bruni O, et al. The spectrum of REM sleep-related episodes in children with type 1 narcolepsy. Brain. (2017) 140:1669-79. doi: 10.1093/brain/awx096

26. Dauvilliers Y, Baumann CR, Carlander B, Bischof M, Blatter T, Lecendreux M, et al. CSF hypocretin-1 levels in narcolepsy, Kleine-Levin syndrome, and other hypersomnias and neurological conditions. J Neurol Neurosurg Psychiatry. (2003) 74:1667-73. doi: 10.1136/jnnp.74.12.1667

27. Vernet C, Leu-Semenescu S, Buzare MA, Arnulf I. Subjective symptoms in idiopathic hypersomnia: beyond excessive sleepiness. J Sleep Res. (2010) 19:525-34. doi: 10.1111/j.1365-2869.2010.00824.x
28. Yang CM, Huang YS, Song YC. Clinical utility of the Chinese version of the pediatric daytime sleepiness scale in children with obstructive sleep apnea syndrome and narcolepsy. Psychiatry Clin Neurosci. (2010) 64:13440. doi: 10.1111/j.1440-1819.2009.02054.X

29. Guo ZN, Sun X, Zhao Y, Yan X, Zhang R, Wang Z, et al. Temporal course of cerebral autoregulation in patients with narcolepsy Type 1: two case reports. Front Neurol. (2019) 9:1155. doi: 10.3389/fneur.2018. 01155

30. Arand DL, Bonnet MH. The multiple sleep latency test. Handb Clin Neurol. (2019) 160:393-403. doi: 10.1016/B978-0-444-64032-1.0 0026-6

Conflict of Interest: The authors declare that the research was conducted in the absence of any commercial or financial relationships that could be construed as a potential conflict of interest.

Copyright (c) 2021 Deng, Zhang, Zhang, Tang, Guo, Lv, Wang and Yang. This is an open-access article distributed under the terms of the Creative Commons Attribution License (CC BY). The use, distribution or reproduction in other forums is permitted, provided the original author(s) and the copyright owner(s) are credited and that the original publication in this journal is cited, in accordance with accepted academic practice. No use, distribution or reproduction is permitted which does not comply with these terms. 